\title{
ORIGINAL RESEARCH \\ Hyperecho-Turbo Spin-Echo Sequences at 3T: Clinical Application in Neuroradiology
}

\author{
R.H. Tetzlaff \\ I. Mader \\ W. Küker \\ J. Weber \\ S. Ziyeh \\ A. Schulze-Bonhage \\ J. Hennig \\ M. Weigel
}

BACKGROUND AND PURPOSE: Hyperecho-turbo spin-echo (hyperTSE) sequences were developed to reduce the specific absorption rate (SAR), especially at high fields such as $3 T$ and above. The purpose of this study was to quantitatively and qualitatively assess the detection of neuroradiologic pathologies by hyperTSE in comparison with standard turbo spin-echo (TSE180 ) sequences.

MATERIALS AND METHODS: TSE $180^{\circ}$ and hyperTSE images with parameters adapted for equal T2 contrast were acquired on a $3 T$ whole-body system in 51 patients with 54 cerebral pathologies. Region-of-interest analysis was performed of signal intensities of pathologies, normal white and gray matter, CSF, and the SD of noise. Signal intensity-to-noise ratios (SNRs) and contrast-to-noise ratios (CNRs) for healthy tissues and pathologies were determined. A qualitative rating concerning artifacts, lesion conspicuity, and image quality was performed by 2 experienced neuroradiologists.

RESULTS: HyperTSE sequences were equivalent to standard TSE $180^{\circ}$ sequences for the CNR of pathologies and of the contrast between gray and white matter. The SNR of gray and white matter and CSF were also the same. The CNRs of the pathologies in hyperTSE and TSE $180^{\circ}$ images were strongly correlated with each other $(r=0.93, P=.001)$. The visual rating of images revealed no significant differences between hyperTSE and TSE $180^{\circ}$.

CONCLUSION: HyperTSE sequences proved to be qualitatively and quantitatively equivalent to TSE $180^{\circ}$ sequences in the detection of high- and low-signal-intensity lesions. They provide equal CNR of pathologies and of gray minus white matter and reduce the imaging restrictions of conventional TSE $180^{\circ}$ imposed by SAR limitations at 3T.
$T^{T}$ he use of standard turbo spin-echo $\left(\mathrm{TSE} 180^{\circ}\right)$ sequences $^{1}$ is well established in clinical routine at lower magnetic fields. At higher fields, the inherent robustness of spin-echo sequences against field inhomogeneities and susceptibility artifacts is even more desirable. Tolerable specific absorption rate (SAR) levels, however, are very soon exceeded by using multiple refocusing pulses with a $180^{\circ}$ flip angle. Common approaches in clinical routine MR imaging to overcome SAR limitations have several disadvantages: Reducing the number of sections keeps the acquisition time constant but compromises volume coverage. Prolonging TR keeps volume coverage unchanged but increases the acquisition time. An overall reduction of the (constant) refocusing flip angle reduces SAR but also markedly reduces signal intensity to noise. Moreover, refocusing flip angles that deviate from $180^{\circ}$ cause $\mathrm{T} 1-$ weighted stimulated echo contributions to participate in echo formation and, thus, lead to a reduced $\mathrm{T} 2$ contrast in the image. $^{2-4}$

The generic hyperecho scheme is a possible solution to this problem because it allows a considerable reduction of SAR while

Received July 30, 2006; accepted after revision December 5, 2007.

From the Department of Neuroradiology (R.H.T., I.M., J.W., S.Z.), Neurocenter of the University Hospital Freiburg, Freiburg, Germany; Department of Radiology (R.H.T.), German Cancer Research Center, Heidelberg, Germany; Department of Neuroradiology (W.K.), John Radcliffe Hospital, Oxford, UK; Institute for Diagnostic Radiology (S.Z.), Freiburg, Germany; Epilepsy Center (A.S.-B.), University Hospital Freiburg, Freiburg, Germany; and Department of Radiology and Medical Physics (J.H., M.W.), University Hospital Freiburg, Freiburg, Germany.

Both R.H. Tetzlaff and I. Mader contributed equally to this paper.

This study was supported by Deutsche Forschungsgemeinschaft (German Research Community) grant HE 1875/14-1.

Please address correspondence to Irina Mader, MD, Department of Neuroradiology, Neurocenter of the University Hospital Freiburg, Breisacher Strasse 64, D-79106 Freiburg, Germany; e-mail: irina.mader@uniklinik-freiburg.de

DOl 10.3174/ajnr.A0971 preserving full signal intensity-to-noise ratios (SNRs). ${ }^{3-6}$ Variable refocusing flip angles are used so that high flip angles produce a high signal for the encoding of the central $k$-space, and lower flip angles are applied for the acquisition of outer parts of the $k$-space. Asymmetric hyperechoes (smooth transitions between pseudosteady states) are the most flexible hyperecho approach, which allows the flip angles to be varied freely for an optimized signalintensity behavior (hyperecho-turbo spin-echo sequences [hyperTSE]) (Fig 1). ${ }^{3,6}$ Due to their low flip angles, hyperTSE sequences also display, however, a reduced $\mathrm{T} 2$ contrast compared with standard $\mathrm{TSE} 180^{\circ}$ sequences with constant $180^{\circ}$ flip angles at a given TE., 3

Recently, it has been shown by calculations, simulations, and a thorough volunteer study that a defined prolongation of TE can compensate for this reduced T2 contrast in hyperTSE. ${ }^{3,4}$ Thus, hyperTSE sequences are highly suitable for low SAR imaging of volunteers with full SNR and equal T2contrast as TSE $180^{\circ}$.

The purpose of this study was to assess quantitatively the application of such T2 contrast-adapted hyperTSE sequences in comparison with the corresponding T2-weighted standard $\mathrm{TSE} 180^{\circ}$ sequences, regarding contrast-to-noise ratios (CNRs) for the pathologies and for gray-white matter contrast, in patients with neurologic or neurosurgical diseases. Furthermore, a qualitative rating of the images presented in random order to 2 experienced neuroradiologists blinded to the methods was intended to provide a subjective visual assessment.

\section{Methods}

The study was approved by the local ethics review board and written informed consent was obtained from all patients.

\section{Subjects}

Subjects were selected among clinically stable outpatients and inpatients, especially if a relevant diagnostic benefit was expected from 

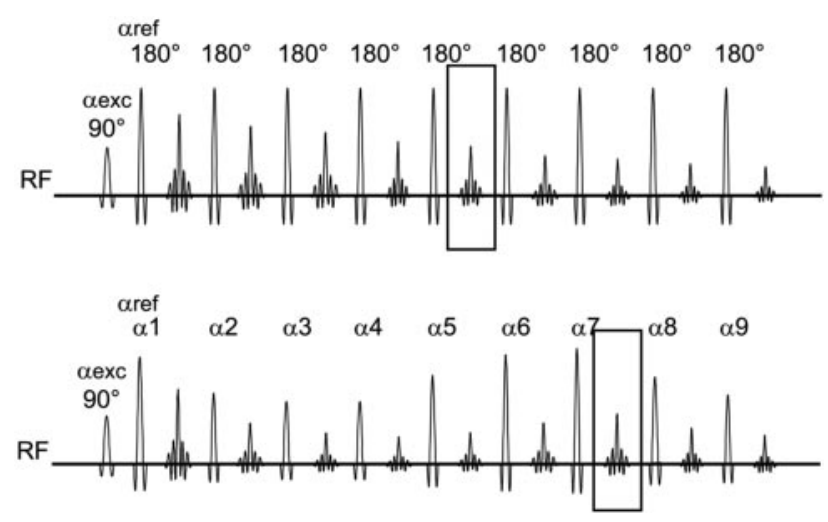

Fig 1. The basic principle of TSE $180^{\circ}$ and hyperTSE. A sketch of the radio-frequency (RF) of the refocusing pulses applying flip angles $\alpha_{\text {ref }}$ is shown with time in TSE180 (upper graph) and hyperTSE (lower graph). In TSE180 , a constant $\alpha_{\text {ref }}=180^{\circ}$ is used, whereas in hyperTSE, $\alpha_{\text {ref }}$ is varied along the echo train. The box indicates the echo written in the center of the $k$-space, which corresponds to the respective TE. Note that the hyperTSE uses a substantial later echo than the TSE180 ${ }^{\circ} \alpha_{\text {exec }}$ indicates excitation pulse.

high-resolution imaging — for example, in epilepsy or when an additional MR angiography was intended. A total of 51 patients (30 males, 21 females) with 54 pathologies were included. The spectrum of pathologies consisted of 1 hemangioblastoma, 2 pilocytic astrocytomas, 1 ganglioglioma, 1 anaplastic astrocytoma, 3 cavernomas, 2 hemorrhagic metastases, 14 focal cortical dysplasias, 14 hippocampal scleroses, 3 cases of encephalomyelitis disseminata, 1 biotin responsive basal ganglia disease, 1 periventricular leucomalacia, 1 Canavan disease, 1 recent and 1 old infarction, 1 residual of intracerebral hemorrhage, 1 cerebellar dysplasia, 1 focal gliosis, 1 unspecific white matter lesion, 2 contusional lesions, 1 malrotation of the hippocampus, and 1 hypothalamic hamartoma. The median age was 32 years; the age range was 4 through 70 years. Diagnosis was established by open or stereotactic operation, follow-up, additional nonimaging investigations, or a second opinion of an external specialist in the field (S.Z. and A.S.-B.).

\section{MR Imaging}

Imaging was performed on a 3T whole-body system (Magnetom Trio; Siemens Medical Systems, Erlangen, Germany) by using the built-in body coil for transmission and a standard circularly polarized head coil for reception.

A hyperTSE sequence $\left(\mathrm{TR}=5200 \mathrm{~ms}, \mathrm{TE}=130 \mathrm{~ms}, \mathrm{TE}_{\text {eff }}=101\right.$ $\mathrm{ms}$, no intersection gap, echo-train length $=17$, in-plane resolution $=0.6 \times 0.4 \mathrm{~mm}$, section thickness $=2 \mathrm{~mm}$ in 44 patients and 5 $\mathrm{mm}$ in 7 patients, 38 sections, 2 concatenations, 2 averages, total acquisition time $=8: 10$ minutes) was performed with section orientation adapted to the suspected location of pathology. The hyperTSE used varied flip angles in the range between $60^{\circ}$ and $180^{\circ}$ by means of sinusoidal ramps, ${ }^{4}$ and the hyperTSE was identical for all experiments.

Identically centered and oriented standard TSE $180^{\circ}$ sequences $\left(\mathrm{TR}=5200 \mathrm{~ms}, \mathrm{TE}=100 \mathrm{~ms}, \mathrm{TE}_{\text {eff }}=100 \mathrm{~ms}\right.$, no intersection gap, echo-train length $=17$, in-plane resolution $=0.6 \mathrm{~mm} \times 0.4 \mathrm{~mm}$, section thickness $=2 \mathrm{~mm}$ in 44 patients and $5 \mathrm{~mm}$ in 7 patients, 17 sections, 2 concatenations, 2 averages, total acquisition time $=$ 8:10 minutes) were performed. Image sets were transferred to a PACS (JVision 3.3.16; Tiani Medgraph, Vienna, Austria) for analysis.

\section{Matching of T2 Contrast}

T1-weighted stimulated echoes contribute to the echo formation whenever refocusing flip angles deviate from $180^{\circ}$ in a turbo/fast spinecho train with constant or varying flip angles. Hence, the image contrast changes. ${ }^{3,4,7,8}$ Recently, a theoretic framework for quantitative contrast characterization and adaptation was developed, being verified by numeric simulations and a thorough volunteer study., 3,8 It was shown that a $\mathrm{TE}_{\text {eff }}$ can be determined that accurately describes the overall T2 contrast present in such TSE images. ${ }^{4,8}$ This T 2 contrast is reduced compared with standard $\mathrm{TSE} 180^{\circ}$ sequences at a given $\mathrm{TE}$, thus $\mathrm{TE}_{\text {eff }}<\mathrm{TE}^{3,4} \mathrm{TE}_{\text {eff }}$ can be calculated via the extended phase graph concept ${ }^{9}$ and only depends on the selected refocusing flip angles and TE. ${ }^{4,8}$ The contrast framework was implemented such that the current $\mathrm{TE}_{\mathrm{eff}}$ of the used hyperTSE was displayed in the protocol editor of the scanner. Before the whole study, a hyperTSE with imaging parameters identical to the standard $\operatorname{TSE} 180^{\circ}$ was created. $\mathrm{TE}_{\text {hyperTSE }}$ was then increased until its $\mathrm{TE}_{\text {eff }}$ closely matched the $\mathrm{TE}$ of the standard $\mathrm{TSE} 180^{\circ}$ : $\mathrm{TE}_{\text {eff-hyperTSE }} \cong \mathrm{TE}_{\mathrm{TSE} 180^{\circ}}$. For our applied protocol, this meant a prolonged $\mathrm{TE}_{\text {hyperTSE }}=130 \mathrm{~ms}$ that leads to a $\mathrm{TE}_{\text {eff-hyperTSE }}=101 \mathrm{~ms}$, which almost coincides with $\mathrm{TE}_{\mathrm{TSE}}=$ $\mathrm{TE}_{\text {eff-TSE }}=100 \mathrm{~ms}$.

\section{Quantitative Evaluation}

For each pair of sequences (hyperTSE and TSE180 $0^{\circ}$ ), a region-ofinterest analysis was performed by an experienced neuroradiologist (I.M.) to obtain the signal intensities of the pathologies. The size of the region of interest of the pathologies was variable and adapted to the size of the pathology but was kept identical for hyperTSE and $\mathrm{TSE} 180^{\circ}$. The regions of interest for gray matter (average, $\sim 7 \mathrm{~mm}^{2}$ ) were obtained from frontal and temporal gray matter. In 1 patient, no normal gray matter could be obtained. The region of interest of white matter (average, $\sim 20 \mathrm{~mm}^{2}$ ) was obtained in the genu of the corpus callosum; and that of CSF (average, $\sim 9 \mathrm{~mm}^{2}$ ), from the frontal horn of the side ventricle. An additional region of interest analysis was performed to obtain the SD of noise by using a region of interest of $\sim 5$ $\mathrm{cm}^{2}$ (Fig 2). The SNRs of pathologies, gray matter, and white matter were calculated as

1)

$$
\mathrm{SNR}_{\text {tissue }}=\mathrm{SI}_{\text {tissue }} /\left(1.53^{\star} \mathrm{SD}_{\text {noise }}\right) .
$$

The CNRs of pathologies were calculated as

2) $\mathrm{CNR}_{\text {pathology }}=\left[\mathrm{SI}_{\text {pathology }}-\left(\right.\right.$ mean $\left.\left.\mathrm{SI}_{\text {gray \& white matter }}\right)\right] /$

$$
\left(1.53^{\star} \mathrm{SD}_{\text {noise }}\right) \text {. }
$$

The term "mean signal intensity $[\mathrm{SI}]_{\text {gray \& white matter" was chosen }}$ because the pathologies often involved both gray and white matter or the gray-white matter border. The mean SI of gray and white matter should account for both tissues.

The CNRs of normal gray and white matter were calculated as

$$
\text { 3) } \mathrm{CNR}_{\text {gray }} \text { - white matter }=\left(\mathrm{SI}_{\text {gray matter }}-\mathrm{SI}_{\text {white matter }}\right) /
$$

$$
\left(1.53 * \mathrm{SD}_{\text {noise }}\right) .
$$

The additional factor of $1 / 1.53$ in equations $1-3$ accounts for the fact that magnitude images were evaluated, thus the original Gaussian distributed noise transforms to a Rician distributed noise. ${ }^{10}$

\section{Visual Rating}

Corresponding sections representative of the respective pathology were selected from each patient and presented side by side in randomized order to 2 experienced neuroradiologists (W.K., J.W.). These 

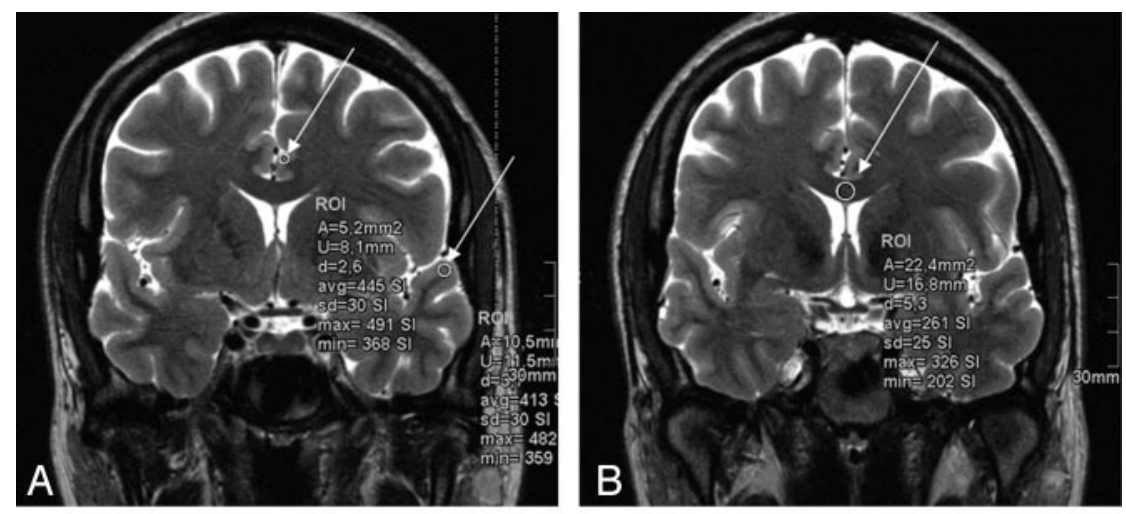

Fig 2. Representative regions of interest. $A$, Regions of interest for gray matter obtained from the frontal and temporal lobes (arrows). An average of the measurements was calculated. $B$, Region of interest for white matter obtained from the genu of the corpus callosum (arrow). $C$, Region of interest for CSF obtained from the frontal horn of the left ventricle (arrow). D, Region of interest for the SD of noise obtained from the noise in the upper left quadrant of the image.
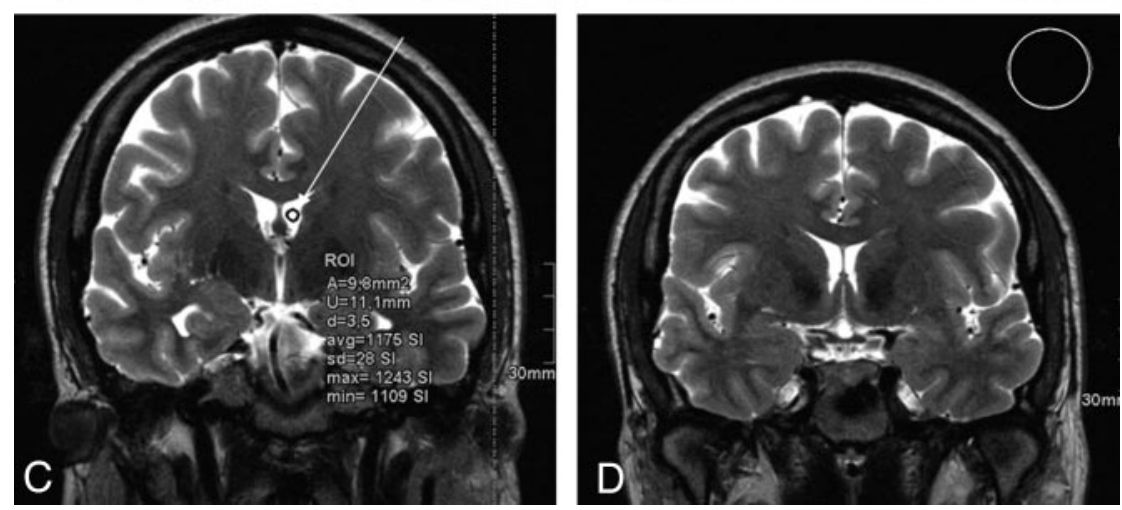

images were rated on a scale of $1-5$ for the presence of motion and flow artifacts, lesion conspicuity, and a subjective appraisal of overall image quality.

\section{Statistics}

Statistical evaluation was performed with the Statistical Package for the Social Sciences, Version 15.0 (SPSS, Chicago, Ill).

A normal distribution was found for the $\mathrm{CNR}_{\text {pathology }}$ and

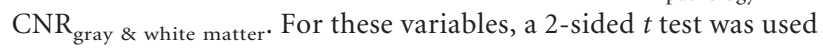
to compare hyperTSE and TSE $180^{\circ}$. A Pearson correlation coefficient was calculated for the $\mathrm{CNR}_{\text {pathology }}$ in hyperTSE and TSE $180^{\circ}$. The SNRs of gray and white matter and CSF were not normally distributed, thus a Wilcoxon signed rank test for comparison of hyperTSE and TSE $180^{\circ}$ was applied. Subjective rankings were also tested for the difference between TSE and TSE $180^{\circ}$ by a Wilcoxon signed rank test. Bonferroni correction adjusting for multiple testing was performed. A $P$ value $<.05$ was considered significant.

\section{Results}

HyperTSE sequences were equivalent to standard TSE $180^{\circ}$ sequences in the detection of all pathologies. There was no significant difference in the SNR of gray matter, white matter, and CSF between hyperTSE and TSE $180^{\circ}$. Also, no significant difference was found between hyperTSE and TSE180 for $\mathrm{CNR}_{\text {gray }}$ - white matter and $\mathrm{CNR}_{\text {pathology, }}$ the latter given in Table 1. The CNR of the pathologies in hyperTSE correlated well with the CNR in standard TSE180 $0^{\circ}(r=0.93, P=.001)$ (Fig 3). Visual ratings were not significantly different for the presence of motion and flow artifacts, lesion conspicuity, and subjective appraisal of image quality (Table 2 ).

Representative cases, including 2 hyperintense lesions (a posttraumatic contusional state and a hippocampal sclerosis) and 1 hypointense lesion (cavernoma), are shown in Figs 4-6, respectively.

\section{Discussion}

In several volunteer studies, hyperTSE sequences have been proved to produce high-quality T2-weighted images at a drastically reduced SAR of typically $>65 \% .^{3-6}$ The purpose of this study was a clinical evaluation of $\mathrm{T} 2$ contrast-adapted hyperTSE in comparison with standard TSE $180^{\circ}$ regarding detection of pathologies, a quantitative assessment of SNR and $\mathrm{CNR}$, and a subjective assessment of image quality. High-resolution images with thin sections are especially necessary in neuroradiologic pathologies. Consequently, a large number of

\begin{tabular}{|c|c|c|c|c|c|c|}
\hline Pathology & Tumor & Hippocampal Sclerosis & FCD & ED & Neuropediatric & Misc. \\
\hline No. of Pathologies & 5 & 12 & 15 & 3 & 3 & 16 \\
\hline \multicolumn{7}{|c|}{$\mathrm{C} / \mathrm{N}$ of pathology in hyperTSE } \\
\hline Mean & 22.7 & 8.0 & 6.6 & 15.6 & 14.0 & 11.0 \\
\hline SD & 13.6 & 4.3 & 7.1 & 17.1 & 8.2 & 11.0 \\
\hline \multicolumn{7}{|c|}{$\mathrm{C} / \mathrm{N}$ of pathology in TSE $180^{\circ}$} \\
\hline Mean & 16.9 & 7.9 & 5.9 & 11.9 & 13.3 & 9.5 \\
\hline SD & 17.4 & 4.5 & 5.8 & 13.4 & 11.4 & 11.7 \\
\hline
\end{tabular}

Note:- C/N indicates contrast-to-noise ratio; SD, standard deviation; FCD, focal cortical dysplasia; ED, encephalomyelitis disseminata; Misc., miscellaneous. 


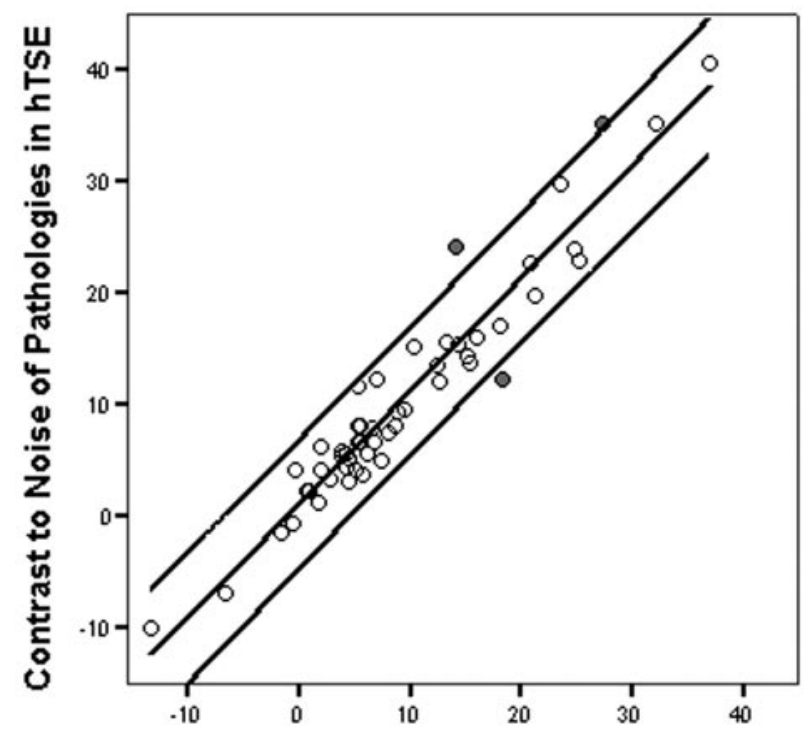

Contrast to Noise of Pathologies in TSE $180^{\circ}$

Fig 3. Linear regression analysis and $95 \%$ predictive interval of single values between contrast to noise of pathology in hyperTSE and $\operatorname{TSE} 180^{\circ}(r=0.93, P=.001)$. Three outliers are marked in gray. hTSE indicates hyperTSE.

sections must be acquired for volume coverage of the entire brain, and long TEs are used to keep the acquisition time tolerable for patients. Both methods result in a high radio-frequency power deposition so that SAR limitations are easily exceeded. The standard TSE $180^{\circ}$ was used in our protocol (eg, operated at the maximal SAR limit allowed by the scanner). Thus, only 17 sections could be acquired, leading to a poor brain coverage and severely hindering an overall diagnosis. Because the applied hyperTSE had an SAR saving of $68 \%$, this tremendous additional reserve in SAR was translated into the acquisition of 38 sections, sufficient for a complete coverage. The residual reserve in SAR of the hyperTSE could have been invested in an even longer TE length to speed up acquisition. However, this was not performed, to ensure comparability between hyperTSE and standard TSE $180^{\circ}$.

The use of low-refocusing flip angles in generic TSE sequences causes T1-weighted stimulated echoes to participate in signalintensity formation. ${ }^{2-4}$ These $\mathrm{T} 1$ contributions lead to a pronounced prolongation of the signal-intensity decay and, thus, to a reduced T2 contrast. ${ }^{3,4}$ For a TSE with constant $120^{\circ}$ refocusing flip angles, for example, T1 contributions are approximately $12 \%$; and for the used hyperTSE, they are approximately $30 \%{ }^{4}$ This directly results, compared with an equivalent standard $\mathrm{TSE} 180^{\circ}$, in a reduced $\mathrm{T} 2$ contrast by the same amount of $12 \%$ and 30\%, respectively. Because the T2 contrast is essential for diagnosis, the published framework for T2 contrast adaptation was used to compensate for this effect. ${ }^{3,4}$ Prolonging TE $\mathrm{hyperTSE}$ from $100 \mathrm{~ms}$ to $130 \mathrm{~ms}$ (ie, a corresponding compensation of $30 \%$ in TE) reinstated the same effective T2 weighting. In Figs $4-6$, the framework, validated in a thorough volunteer study, ${ }^{4}$ also works very well in our patient study.

Besides the diagnostic value of sustained T2 contrast, the contrast adaptation was also mandatory for a quantitative comparison of SNR and CNR values between hyperTSE and standard TSE $180^{\circ}$ in our study. The quantitative equivalence of hyperTSE and TSE $180^{\circ}$ is demonstrated by the strong correlation of $\mathrm{CNR}_{\text {pathology. }}$ In particular, all hyperintense lesions in TSE $180^{\circ}$ were indicated as hyperintense lesions in hyperTSE. Hypointense lesions (hemosiderin rim in 3 cavernomas and in an old hemorrhage) were also hypointense in both sequences (Fig 6). Additional artifacts and a degraded point spread function (ie, "blurring") were not noted. ${ }^{11}$

Besides the general very good contrast adaptation between TSE $180^{\circ}$ and hyperTSE observed in the study, 3 outliers can be noted, which were marked gray in Fig 3. These outliers are attributed to motion artifacts in 2 cases and to 1 discreet shift of the head, compared with a technically identical section position, combined with slight motion and phase artifacts being only visible with a narrow contrast window. In all 3 cases, the measurement of the SD of noise was compromised by the motion artifacts. In the case of the slightly different section position, different partial volume effects may account for the difference in CNR.

By subjective inspection, however, the difference of $\mathrm{CNR}_{\text {pathology }}$ would not have been depicted. This is corroborated by the results of the subjective rating, in which no significant differences between hyperTSE and TSE $180^{\circ}$ could be found.

As already mentioned, refocusing flip angles that deviate from $180^{\circ}$ contribute a T1-weighting in the signal-intensity formation. Thus, a minor T1 contrast is introduced in the image, which is visible to the trained eye. Apart from this qualitative statement, the additional induced $\mathrm{T} 1$ contrast did not affect $\mathrm{CNR}_{\text {pathology }}$ in hyperTSE and TSE180 $0^{\circ}$. The equal $\mathrm{CNR}_{\text {pathology }}$ in both indicates an equivalence of the sequences.

The negligible influence of $\mathrm{T} 1$ weighting on the $\mathrm{CNR}$ values can also be quantitatively understood: Because the TE is considerably lower than the T1 relaxation times of the observed tissues, only minor $\mathrm{T} 1$ decay can take place.

In addition to their $\mathrm{T} 1$ and $\mathrm{T} 2$ behavior, $\mathrm{TSE} 180^{\circ}$ and hyperTSE can be expected to differ with respect to signal-intensity attenuation by magnetization transfer and to motional effects, both on a microscopic scale $\left(\mathrm{T} 2^{\prime}\right)$ and due to vascular flow. ${ }^{2,3,12-17}$ Thus, differences in signal-intensity behavior could have been expected despite the matched $\mathrm{T} 2$ contrast used in both sequences. The equal SNR and CNR measured in this study show that these mechanisms do not play a relevant role in neuroradiologic imaging.

\begin{tabular}{|c|c|c|c|c|c|c|}
\hline \multirow[t]{2}{*}{ Rater } & \multicolumn{2}{|c|}{ Artifacts } & \multicolumn{2}{|c|}{ Lesion Conspicuity } & \multicolumn{2}{|c|}{ Quality } \\
\hline & hyperTSE & TSE $180^{\circ}$ & hyperTSE & TSE $180^{\circ}$ & hyperTSE & TSE $180^{\circ}$ \\
\hline 1 & $4.50 / 5 / 0.61$ & $4.46 / 5 / 0.64$ & $4.51 / 5 / 0.64$ & $4.44 / 5 / 0.69$ & $4.19 / 4 / 0.48$ & $4.11 / 4 / 0.60$ \\
\hline 2 & $4.59 / 5 / 0.74$ & $4.65 / 5 / 0.70$ & $4.17 / 5 / 1.09$ & $4.09 / 51.25$ & $4.04 / 4 / 0.85$ & $3.98 / 4 / 0.90$ \\
\hline
\end{tabular}

${ }^{*}$ Given are mean/median/SD of a 5-point scoring scale within a range of 1 (poor) through 5 (excellent). 

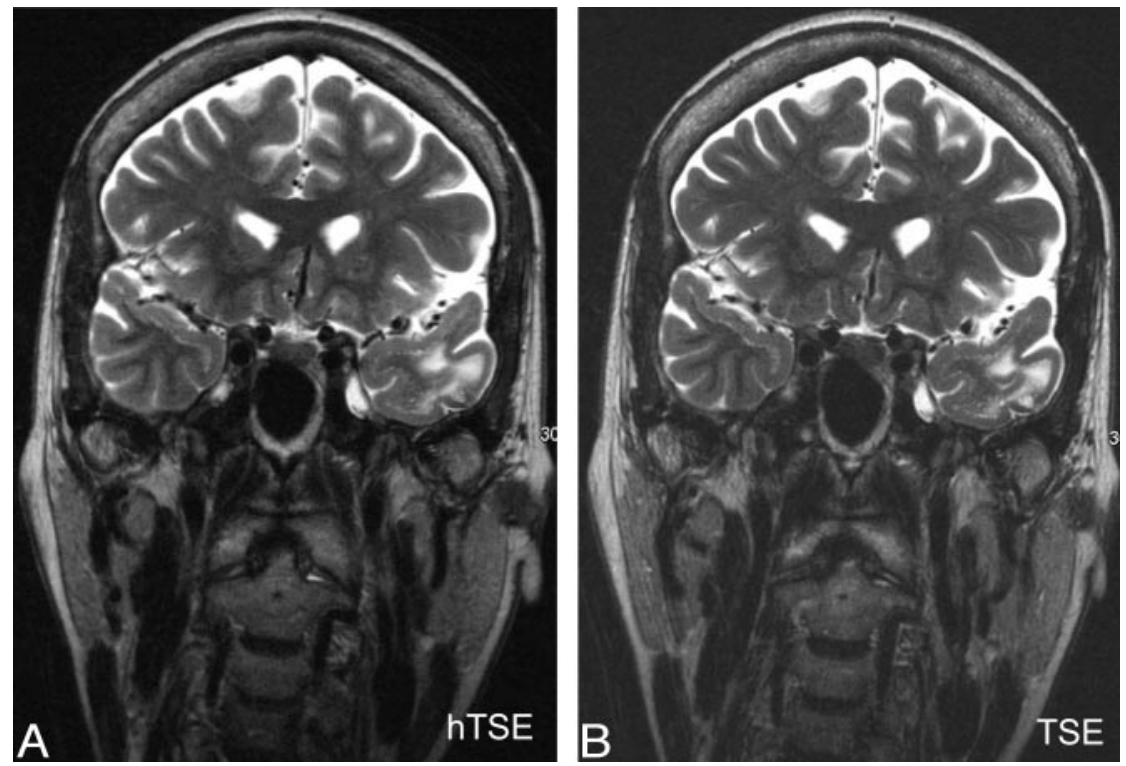

Fig 4. $A$, hyperTSE (hTSE). $B$, TSE $180^{\circ}$. Note the gliosis in the white and gray matter of the left temporal lobe after trauma. In both sequences, the hyperintense findings are clearly visible.
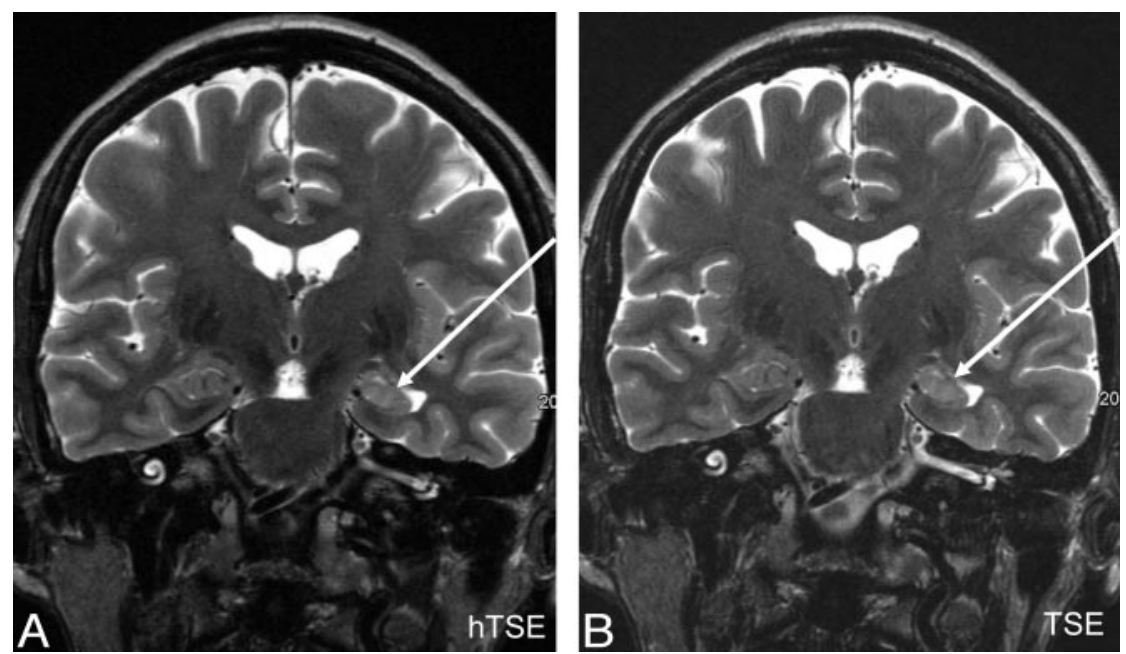

Fig 5. $A$, hyperTSE (hTSE). $B$, TSE180 . The left hippocampus is atrophic and has increased signal intensity attributed to hippocampal sclerosis (arrows). This finding was confirmed by surgery. The right hippocampus shows the typical dentation, and the particular layers are visible on both sequences.
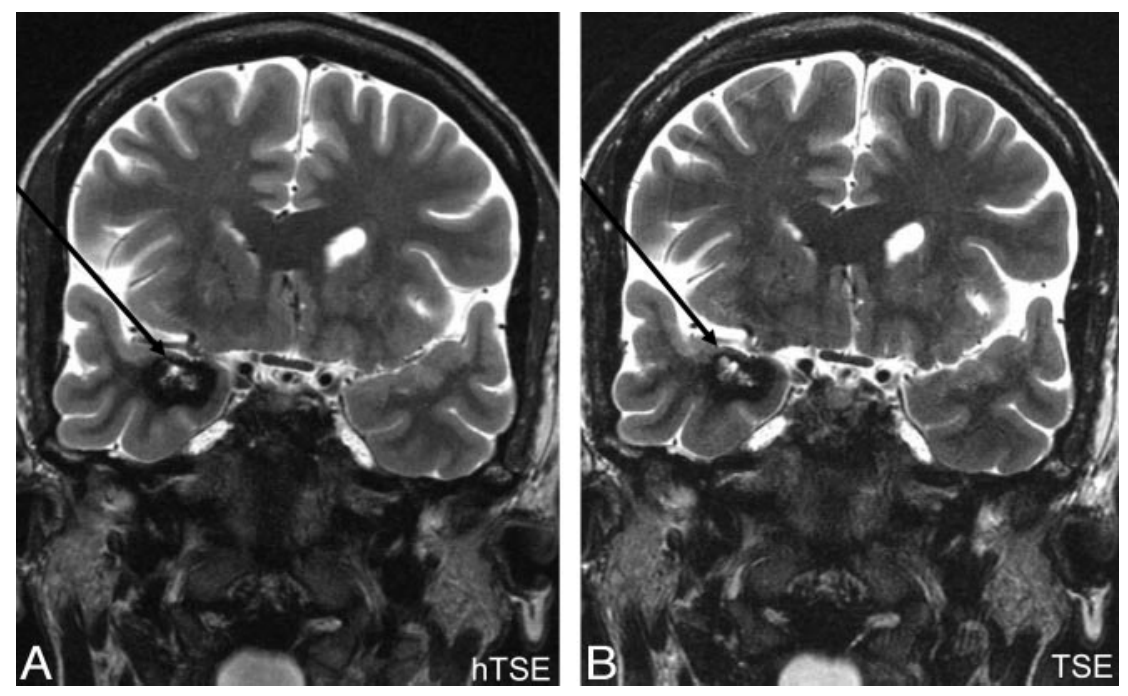

Fig 6. A, HyperTSE (hTSE). B, TSE $180^{\circ}$. A right mesiotemporal cavernoma (arrows) consisting of a hypointense hemosiderin rim and a central hyperintense matrix containing methemoglobin is clearly depicted in both sequences. It is important that both parts of blood degradation products are depicted; this depiction was successful for both sequences. The diagnosis was confirmed by surgery.

\section{Conclusion}

T2 contrast-adapted hyperTSE sequences are equivalent to standard TSE $180^{\circ}$ sequences in the detection of high- and low-signal-intensity lesions. They provide similar CNRs of gray minus white matter and pathology. Subjective evalua- tion additionally corroborates the equivalency of both sequences. Thus, hyperTSE sequences can be applied at $3 \mathrm{~T}$ with an equivalent T2 contrast to standard TSE $180^{\circ}$ and a full brain coverage without exceeding the SAR limits. 


\section{References}

1. Hennig J, Nauerth A, Friedburg H. RARE imaging: a fast imaging method for clinical MR. Magn Reson Med 1986;3:823-33

2. Constable RT, Anderson AW, Zhong J, et al. Factors influencing contrast in fast spin-echo MR imaging. Magn Reson Imaging 1992;10:497-511

3. Hennig J, Weigel M, Scheffler K. Multiecho sequences with variable refocusing flip angles: optimization of signal behavior using smooth transitions between pseudo steady states (TRAPS). Magn Reson Med 2003;49:527-35

4. Weigel M, Hennig J. Contrast behavior and relaxation effects of conventional and hyperecho-turbo spin echo sequences at 1.5 and 3 T. Magn Reson Med 2006;55:826-35

5. Hennig J, Scheffler K. Hyperechoes. Magn Reson Med 2001;46:6-12

6. Hennig J, Weigel M, Scheffler K. Calculation of flip angles for echo trains with predefined amplitudes with the extended phase graph (EPG)-algorithm: principles and applications to hyperecho and TRAPS sequences. Magn Reson Med 2004;51:68-80

7. Hennig J. Multiecho imaging sequences with low refocusing flip angles. $J$ Magn Reson 1988;78:397-407

8. Weigel M, Zaitsev M, Hennig J. Inversion recovery prepared turbo spin echo sequences with reduced SAR using smooth transitions between pseudo steady states. Magn Reson Med 2007;57:631-37
9. Hennig J. Echoes: how to generate, recognize, use or avoid them in MR-imaging sequences. Concepts in Magnetic Resonance 1991;3:125-43

10. Weisskoff RM. Simple measurement of scanner stability for functional NMR imaging of activation in the brain. Magn Reson Med 1996;36: 643-45

11. Busse RF. Reduced RF power without blurring: correcting for modulation of refocusing flip angle in FSE sequences. Magn Reson Med 2004;51: 1031-37

12. Balaban RS, Ceckler TL. Magnetization transfer contrast in magnetic resonance imaging. Magn Reson Q 1992;8:116-37

13. Duewell S, Wolff SD, Wen $\mathrm{H}$, et al. MR imaging contrast in human brain tissue: assessment and optimization at 4 T. Radiology 1996;199:780-86

14. Henkelman RM, Stanisz GJ, Graham SJ. Magnetization transfer in MRI: a review. NMR Biomed 2001;14:57-64

15. Melki PS, Mulkern RV. Magnetization transfer effects in multislice RARE sequences. Magn Reson Med 1992;24:189-95

16. Ordidge RJ, Gorell JM, Deniau JC, et al. Assessment of relative brain iron concentrations using T2-weighted and T2*-weighted MRI at 3 Tesla. Magn Reson Med 1994;32:335-41

17. Williams CF, Redpath TW, Smith FW. The influence of stimulated echoes on contrast in fast spin-echo imaging. Magn Reson Imaging 1996;14:419-28 